\title{
Common fixed point and coincidence point of generalized contractions in ordered metric spaces
}

Ismat Beg ${ }^{1 *}$ and Asma Rashid Butt ${ }^{2}$

\section{"Correspondence:}

begismat@yahoo.com

${ }^{1}$ Centre for Applicable Mathematics

and Statistics, University of Central

Punjab, Lahore, Pakistan

Full list of author information is

available at the end of the article

\begin{abstract}
Let $(X, \preceq)$ be a partially ordered set and $d$ be a complete metric on $X$. The notion of $f$-contractive for a set-valued mapping due to Latif and Beg is extended through an implicit relation. Coincidence and fixed point results are obtained for mappings satisfying generalized contractions in a partially ordered metric space $X$. Our results improve and extend several known results in the existing literature.
\end{abstract}

MSC: $47 \mathrm{H} 10 ; 47 \mathrm{H} 04 ; 47 \mathrm{H} 07$

Keywords: fixed point; coincidence point; partially ordered set; metric space; set-valued mapping

\section{Introduction and preliminaries}

Let $(X, d)$ be a metric space, $P(X)$ be the class of all non-empty subsets of $X, C l(X)$ be the class of all non-empty closed subsets of $X$ and $C B(X)$ be the class of all non-empty closed and bounded subsets of $X$. For $A, B \in C B(X)$, let

$$
D(A, B):=\max \left\{\sup _{b \in B} d(b, A), \sup _{a \in A} d(a, B)\right\}
$$

where

$$
d(a, B):=\inf _{b \in B} d(a, b)
$$

$D$ is called the Hausdorff metric induced by $d$.

Let $f: X \rightarrow X$ be any single-valued mapping. A mapping $F: X \rightarrow C B(X)$ is $f$-contractive if there exists a real number $\kappa$ with $0<\kappa<1$ such that

$$
D(F x, F y) \leq \kappa d(f x, f y) \quad \text { for all } x, y \in X
$$

By introducing the notion of $f$-contractiveness for set-valued mappings, Kaneko [1] obtained a result which gives the existence of a coincidence point in a metric space. An improved version of Kaneko's result was obtained by Latif and Beg [2]. They proved the following.

\section{Springer}

O 2012 Beg and Butt; licensee Springer. This is an Open Access article distributed under the terms of the Creative Commons Attribution License (http://creativecommons.org/licenses/by/2.0), which permits unrestricted use, distribution, and reproduction in any medium, provided the original work is properly cited. 
Theorem 1.1 [2, Theorem 2.6] Let $f: X \rightarrow X$ be a continuous map with $f(X)$ complete. Suppose that $F: X \rightarrow C B(X)$ is a $f$-contractive map such that $F(X) \subseteq f(X)$. Then there exists $x_{0} \in X$ such that $f x_{0} \in F x_{0}$.

In [3], Kaneko and Sessa proved the following coincidence point result.

Theorem 1.2 [3] Let $(X, d)$ be a complete metric space $f: X \rightarrow X$ and $F: X \rightarrow C B(X)$ be compatible continuous mappings such that $F(X) \subseteq f(X)$ and

$$
D(F x, F y) \leq \kappa \max \{d(f x, f y), d(f x, F x), d(f y, F y), 1 / 2[d(f x, F y)+d(f y, F x)]\}
$$

for all $x, y \in X$, where $0 \leq \kappa<1$.

Then there exists $x_{0} \in X$ such that $f x_{0} \in F x_{0}$.

A mapping $f: X \rightarrow X$ is called $K$-mapping if there exists a real number $\kappa$ with $0 \leq \kappa<\frac{1}{2}$ such that

$$
d(f x, f y) \leq \kappa[d(x, f x)+d(y, f y)] \text { for all } x, y \in X .
$$

Definition 1.3 Let $F: X \rightarrow P(X)$ be a mapping. A point $x \in X$ is said to be a fixed point of $F$ if $x \in F x$.

Definition 1.4 Let $F: X \rightarrow P(X)$ and $f: X \rightarrow X$ be mappings. A point $x \in X$ is said to be a coincidence point of $F$ and $f$ if $f x \in F x$.

Definition 1.5 A partial order is a binary relation $\preceq$ over a set $X$ which satisfies the following conditions:

1. $x \preceq x$ (reflexivity);

2. If $x \preceq y$ and $y \preceq x$, then $x=y$ (antisymmetry);

3. If $x \preceq y$ and $y \preceq z$, then $x \preceq z$ (transitivity)

for all $x, y$ and $z$ in $X$.

A set with a partial order $\preceq$ is called a partially ordered set.

Let $(X, \preceq)$ be a partially ordered set and $x, y \in X$. Elements $x$ and $y$ are said to be comparable elements of $X$ if either $x \preceq y$ or $y \preceq x$.

Recently, there have been so many exciting developments in the field of existence of a fixed point in partially ordered sets (see [4-20] and the references cited therein). This trend was started by Ran and Reurings in [11] where they extended the Banach contraction principle in partially ordered sets with some application to a matrix equation. Ran and Reurings [11] proved the following seminal result.

Theorem 1.6 [11] Let $(X, \preceq)$ be a partially ordered set such that every pair $x, y \in X$ has an upper and lower bound. Let $d$ be a complete metric on $X$ and $f: X \rightarrow X$ be a continuous monotone (either order-preserving or order-reversing) mapping. Suppose that the following conditions hold:

1. There exists $\kappa \in(0,1)$ with

$$
d(f(x), f(y)) \leq \kappa d(x, y) \quad \text { for all } x \leq y .
$$


2. There exists $x_{0} \in X$ with $x_{0} \preceq f x_{0}$ or $f x_{0} \preceq x_{0}$.

Then $f$ is a Picard operator $(P O)$, that is, $f$ has a unique fixed point $x^{*} \in X$ and for each $x \in X$,

$$
\lim _{n \rightarrow \infty} f^{n} x=x^{*}
$$

Theorem 1.6 was further extended and refined in $[7-9,12,15,19]$. These results are hybrid of the two fundamental classical theorems: Banach's fixed point theorem (see [21]) and Tarski's [22] fixed point theorem. Our aim in this paper is to introduce a generalization of $f$-contractiveness through an implicit relation. This implicit relation is then used to obtain the existence of coincidence and common fixed points for a pair of single-valued mapping and set-valued mapping on a partially ordered metric space. In Section 2, we prove a coincidence point theorem where we use an implicit relation only for comparable elements of a partially ordered set $X$. Our result generalizes/extends $[1-3,23,24]$ work to partial ordered sets. Section 3 deals with the existence of a common fixed point by using the notion of $k-\preceq$ set-valued mapping, which improves the results of Latif and Beg [2] to partially ordered sets.

We will make use of the following lemma in the proof of our result in the next section.

Lemma 1.7 [25] Let $A, B \in C B(X)$ and $a \in A$. Then for $\epsilon>0$, there exists an element $b \in B$ such that $d(a, b)<D(A, B)+\epsilon$.

Throughout the next two sections, we take $(X, \preceq)$ as a partially ordered set with a complete metric $d$.

\section{Implicit relation and coincidence points}

Implicit relations in metric spaces have been considered by several authors in connection with solving nonlinear functional equations (see, for instance, $[16-18,26]$ and the references cited therein). First, we give some implicit relations for subsequent use.

Let $R_{+}$be the set of nonnegative real numbers and $\mathcal{T}$ be the set of continuous real-valued functions $T: R_{+}^{6} \rightarrow R$ satisfying the following conditions:

$\mathcal{T}_{1}: T\left(t_{1}, t_{2}, \ldots, t_{6}\right)$ is non-increasing in $t_{2}, t_{3}, \ldots, t_{6}$.

$\mathcal{T}_{2}$ : there exists a real number $\kappa$ with $0<\kappa<1$ and $\varepsilon>0$ such that the inequalities

$$
u \leq w+\epsilon
$$

and

$$
T(w, v, v, u, u+v, 0) \leq 0
$$

imply

$$
w \leq \kappa \nu .
$$

$\mathcal{T}_{3}: T(w, 0,0, v, v, 0) \leq 0$ implies $w \leq \kappa v$.

Next, we give some examples for $T$ satisfying $\mathcal{T}_{1}-\mathcal{T}_{3}$. 
Example 2.1 $T\left(t_{1}, \ldots, t_{6}\right)=t_{1}-\alpha\left(\max \left\{t_{2}, t_{3}, t_{4}, 1 / 2\left(t_{5}+t_{6}\right)\right\}\right)$, where $0<\alpha<1$.

$\mathcal{T}_{1}$ : is obvious.

$\mathcal{T}_{2}$ : Let $u>0$, then choose $\epsilon>0$ such that $\alpha u+\epsilon<u$ (this is possible since $0<\alpha<1$ ). As $T(w, v, v, u, u+v, 0) \leq 0$, therefore $w-\alpha(\max \{u, v\}) \leq 0$. Now let $u \leq w+\epsilon$. If $u \geq v$, then $u \leq \alpha u+\epsilon<u$. Hence a contradiction. Thus $u<v$ and $w \leq \alpha v$. If $u=0$, then $w \leq \alpha v$. Thus $\mathcal{T}_{2}$ is satisfied.

$\mathcal{T}_{3}$ : Since $T(w, 0,0, v, v, 0) \leq 0$, therefore $w-\alpha v \leq 0$. It further implies that $w \leq \alpha v$.

Example 2.2 $T\left(t_{1}, \ldots, t_{6}\right)=t_{1}-\alpha\left(\max \left\{t_{2}, \frac{t_{3}+t_{4}}{2}, \frac{t_{5}+t_{6}}{2}\right\}\right)$, where $0<\alpha<1$.

$\mathcal{T}_{1}:$ is obvious.

$\mathcal{T}_{2}$ : Let $u>0$, then choose $\epsilon>0$ so that $\alpha u+\epsilon<u$. Since $T(w, v, v, u, u+v, 0) \leq 0$, therefore $w-\alpha(\max \{u, v\}) \leq 0$. Now let $u \leq w+\epsilon$. If $u \geq v$, then $u \leq \alpha u+\epsilon<u$. Hence a contradiction. Thus $u<v$ and $w \leq \alpha v$. If $u=0$, then $w \leq \alpha v$. Thus $\mathcal{T}_{2}$ is satisfied.

$\mathcal{T}_{3}$ : Since $T(w, 0,0, v, v, 0) \leq 0$, therefore $w-\frac{\alpha}{2} v \leq 0$. It further implies that $w \leq \frac{\alpha}{2} v \leq \kappa v$.

Example 2.3 $T\left(t_{1}, \ldots, t_{6}\right)=t_{1}-\alpha \max \left\{t_{2}, t_{3}, t_{4}\right\}-(1-\alpha)\left(a t_{5}+b t_{6}\right)$, where $0<\alpha<1,0 \leq$ $a, b<1 / 2$.

$\mathcal{T}_{1}$ : is obvious.

$\mathcal{T}_{2}$ : Let $u>0$, then choose $\epsilon>0$ so that $(\alpha+2 a(1-\alpha)) u+\epsilon<u$ (this is possible since $0<\alpha+$ $2 a(1-\alpha)<1)$. Since $T(w, v, v, u, u+v, 0) \leq 0$, therefore $w-\alpha \max \{u, v\}-(1-\alpha) a(u+v) \leq$ 0 . Now let $u \leq w+\epsilon$. If $u \geq v$, then $u \leq[\alpha+2 a(1-\alpha)] u+\epsilon<u$, hence a contradiction. Thus $u<v$ and $w \leq(\alpha+2 a(1-\alpha)) v$. Thus $\mathcal{T}_{2}$ is satisfied with $\kappa=\alpha+2 a(1-\alpha)$.

$\mathcal{T}_{3}$ : Since $T(w, 0,0, v, v, 0) \leq 0$, therefore $w-\alpha v-(1-\alpha) a v \leq 0$. It further implies that $w \leq$ $[\alpha+a(1-\alpha)] v \leq \kappa v$.

Theorem 2.4 Let $f: X \rightarrow X$ and $F: X \rightarrow C B(X)$ satisfying

$$
T(D(F x, F y), d(f x, f y), d(f x, F x), d(f y, F y), d(f x, F y), d(f y, F x)) \leq 0
$$

for all comparable elements $x, y$ of $X$ and for some $T \in \mathcal{T}$. If the following conditions are satisfied:

1. $F(X) \subseteq f(X)$ and $f(X)$ is closed;

2. If $f q \in F x$, then $x \preceq q$;

3. If $y_{n} \in F x_{n}$ is such that $y_{n} \rightarrow y=f p$, then $x_{n} \preceq p$ for all $n$, then there exists $p$ with $f p \in F p$.

Proof Let $x_{0} \in X$, then by using assumptions 1 and 2 , we can choose $x_{1} \in X$ with $x_{0} \preceq x_{1}$ such that $y_{0}=f x_{1} \in F x_{0}$. Since $y_{0} \in F x_{0}$, then for any $\varepsilon>0$, from Lemma 1.7, there exists $y_{1} \in F x_{1}$ such that

$$
d\left(y_{0}, y_{1}\right) \leq D\left(F x_{0}, F x_{1}\right)+\varepsilon
$$

Using assumptions 1 and 2 , since $y_{1} \in F x_{1} \subseteq f(X)$, there exists $x_{2} \in X$ such that $y_{1}=f x_{2} \in$ $F x_{1}$ and so $x_{1} \preceq x_{2}$. 
Now using (A), we have

$$
T\left(D\left(F x_{0}, F x_{1}\right), d\left(f x_{0}, f x_{1}\right), d\left(f x_{0}, F x_{0}\right), d\left(f x_{1}, F x_{1}\right), d\left(f x_{0}, F x_{1}\right), d\left(f x_{1}, F x_{0}\right)\right) \leq 0 .
$$

Using the facts that $d\left(f x_{0}, F x_{0}\right) \leq d\left(f x_{0}, y_{0}\right), d\left(f x_{1}, F x_{1}\right) \leq d\left(y_{0}, y_{1}\right)$,

$$
d\left(f x_{0}, F x_{1}\right) \leq d\left(f x_{0}, y_{1}\right) \leq d\left(f x_{0}, y_{0}\right)+d\left(y_{0}, y_{1}\right), d\left(f x_{1}, F\left(x_{0}\right)\right) \leq d\left(y_{0}, y_{0}\right),
$$

and by $\mathcal{T}_{1}$, we have

$$
T\left(D\left(F x_{0}, F x_{1}\right), d\left(f x_{0}, y_{0}\right), d\left(f x_{0}, y_{0}\right), d\left(y_{0}, y_{1}\right), d\left(f x_{0}, y_{0}\right)+d\left(y_{0}, y_{1}\right), 0\right) \leq 0
$$

that is,

$$
T(w, v, v, u, u+v, 0) \leq 0,
$$

where $w=D\left(F x_{0}, F x_{1}\right), v=d\left(f x_{0}, y_{0}\right), u=d\left(y_{0}, y_{1}\right)$. By using $\mathcal{T}_{2}$, we have $(w \leq \kappa v)$,

$$
D\left(F x_{0}, F x_{1}\right) \leq \kappa d\left(f x_{0}, y_{0}\right)
$$

Using (2.2) in (2.1), we have

$$
d\left(y_{0}, y_{1}\right) \leq \kappa d\left(f x_{0}, y_{0}\right)+\epsilon
$$

Since $y_{1} \in F x_{1}$, then for $\kappa>0$, from Lemma 1.7, there exists $y_{2} \in F x_{2}$ such that

$$
d\left(y_{1}, y_{2}\right) \leq D\left(F x_{1}, F x_{2}\right)+\kappa
$$

Using assumptions 1 and 2, since $y_{2} \in F x_{2} \subseteq f(X)$, there exists $x_{3} \in X$ such that $y_{2}=f x_{3} \in$ $F x_{2}$ and so $x_{2} \preceq x_{3}$.

Now, since $x_{1} \preceq x_{2}$, by using (A), we have

$$
T\left(D\left(F x_{1}, F x_{2}\right), d\left(f x_{1}, f x_{2}\right), d\left(f x_{1}, F x_{1}\right), d\left(f x_{2}, F x_{2}\right), d\left(f x_{1}, F x_{2}\right), d\left(f x_{2}, F x_{1}\right)\right) \leq 0,
$$

by $\mathcal{T}_{1}$ we have

$$
T\left(D\left(F x_{1}, F x_{2}\right), d\left(y_{0}, y_{1}\right), d\left(y_{0}, y_{1}\right), d\left(y_{1}, y_{2}\right), d\left(y_{0}, y_{1}\right)+d\left(y_{1}, y_{2}\right), 0\right) \leq 0
$$

that is

$$
T(w, v, v, u, u+v, 0) \leq 0,
$$

where $w=D\left(F x_{1}, F x_{2}\right), v=d\left(y_{0}, y_{1}\right), u=d\left(y_{1}, y_{2}\right)$. Therefore, by using $\mathcal{T}_{2}$, we have $(w \leq \kappa v)$

$$
D\left(F x_{1}, F x_{2}\right) \leq \kappa d\left(y_{0}, y_{1}\right)
$$

And so from (2.3) and (2.4), we have

$$
d\left(y_{1}, y_{2}\right) \leq D\left(F x_{1}, F x_{2}\right)+\kappa \leq \kappa d\left(y_{0}, y_{1}\right)+\kappa
$$


Again, since $y_{2} \in F x_{2}$, then for $\kappa>0$, from Lemma 1.7, there exists $y_{3} \in F x_{3}$ such that

$$
d\left(y_{2}, y_{3}\right) \leq D\left(F x_{2}, F x_{3}\right)+\kappa^{2}
$$

Using assumptions 1 and 2, since $y_{3} \in F x_{3} \subseteq f(X)$, there exists $x_{4} \in X$ such that $y_{3}=f x_{4} \in$ $F x_{3}$ and so $x_{3} \preceq x_{4}$.

Now, since $x_{2} \preceq x_{3}$, by using (A) we have

$$
T\left(D\left(F x_{2}, F x_{3}\right), d\left(f x_{2}, f x_{3}\right), d\left(f x_{2}, F x_{2}\right), d\left(f x_{3}, F x_{3}\right), d\left(f x_{2}, F x_{3}\right), d\left(f x_{3}, F x_{2}\right)\right) \leq 0,
$$

by $\mathcal{T}_{1}$ we have

$$
T\left(D\left(F x_{2}, F x_{3}\right), d\left(y_{1}, y_{2}\right), d\left(y_{1}, y_{2}\right), d\left(y_{2}, y_{3}\right), d\left(y_{1}, y_{2}\right)+d\left(y_{2}, y_{3}\right), 0\right) \leq 0,
$$

that is

$$
T(w, v, v, u, u+v, 0) \leq 0,
$$

where $w=D\left(F x_{2}, F x_{3}\right), v=d\left(y_{1}, y_{2}\right), u=d\left(y_{2}, y_{3}\right)$. Therefore, by using $\mathcal{T}_{2}$, we have $(w \leq \kappa v)$

$$
D\left(F x_{2}, F x_{3}\right) \leq \kappa d\left(y_{1}, y_{2}\right)
$$

Now, using (2.5), (2.6) and (2.7), we have

$$
d\left(y_{2}, y_{3}\right) \leq \kappa d\left(y_{1}, y_{2}\right)+\kappa^{2} \leq \kappa^{2} d\left(y_{0}, y_{1}\right)+2 \kappa^{2} \text {. }
$$

Continuing in this way, we obtain a sequence $\left\{x_{n}\right\}$ with $x_{n} \preceq x_{n+1}$ such that $y_{n}=f x_{n+1} \in F x_{n}$ for $n \geq 0$ and

$$
\begin{aligned}
& d\left(y_{n}, y_{n+1}\right)<\kappa^{n} d\left(y_{0}, y_{1}\right)+n \kappa^{n}, \\
& \sum_{n=1}^{\infty} d\left(y_{n}, y_{n+1}\right)<d\left(y_{0}, y_{1}\right) \sum_{n=1}^{\infty} \kappa^{n}+\sum_{n=1}^{\infty} n \kappa^{n}<\infty .
\end{aligned}
$$

Hence, $\left\{y_{n}\right\}$ is a Cauchy sequence. So, there exists a point $y$ in the complete metric space $X$ such that

$$
\lim _{n \rightarrow \infty} y_{n}=\lim _{n \rightarrow \infty} f\left(x_{n+1}\right)=y \in \lim _{n \rightarrow \infty} F\left(x_{n}\right) .
$$

Now, since $f(X)$ is closed, there exists $p \in X$ such that $y=f p \in f(X)$ and by assumption 3, $x_{n} \preceq p$ for all $n$.

Now, using (A), we have

$$
T\left(D\left(F x_{n}, F p\right), d\left(f x_{n}, f p\right), d\left(f x_{n}, F x_{n}\right), d(f p, F p), d\left(f x_{n}, F p\right), d\left(f p, F x_{n}\right)\right) \leq 0 .
$$

Now, taking limit as $n \rightarrow \infty$ and using $\mathcal{T}_{2}$, also the facts that $d\left(f x_{n}, f p\right)=d\left(y_{n-1}, y\right) \rightarrow 0$, $d\left(f x_{n}, F x_{n}\right) \leq d\left(y_{n-1}, y_{n}\right) \rightarrow 0, d\left(f p, F x_{n}\right) \leq d\left(y, y_{n}\right) \rightarrow 0$, we have

$$
T\left(\lim _{n \rightarrow \infty} D\left(F x_{n}, F p\right), 0,0, d(y, F p), d(y, F p), 0\right) \leq 0
$$


that is,

$$
T(w, 0,0, v, v, 0) .
$$

By using $\mathcal{T}_{3}$, we get

$$
\lim _{n \rightarrow \infty} D\left(F x_{n}, F p\right) \leq \kappa(d(y, F p)) .
$$

Next, since $y_{n} \in F x_{n}$,

$$
d\left(y_{n}, F v\right) \leq D\left(F x_{n}, F p\right)
$$

taking limit as $n \rightarrow \infty$, we obtain

$$
d(y, F p) \leq \lim _{n \rightarrow \infty} D\left(F x_{n}, F p\right) \leq \kappa d(y, F p)
$$

From above, we have $\kappa>1$, a contradiction. So, $d(y, F p)=0$. Therefore, $d(y, F p)=0$ and $f p=y \in \overline{F p}=F p$.

Remark 2.5 In assumptions 2 and 3 of Theorem 2.4, we need only comparability of the elements. Theorem 2.4 with Example 2.2 partially improve [27, Theorem 3.10].

Corollary 2.6 Let $f: X \rightarrow X$ and $F: X \rightarrow C B(X)$ satisfy

$$
D(F x, F y) \leq \kappa d(f x, f y)
$$

for some $\kappa$ with $0<\kappa<1$ and for all comparable elements $x, y$ of $X$.

Also, assume that the following conditions are satisfied:

1. $F(X) \subseteq f(X)$ and $f(X)$ is closed.

2. If $f \in F x$, then $x \preceq q$.

3. If $y_{n} \in F x_{n}$ is such that $y_{n} \rightarrow y=f p$, then $x_{n} \preceq p$ for all $n$.

Then there exists $p$ such that $f p \in F p$.

Proof Let $T\left(t_{1}, \ldots, t_{6}\right):=t_{1}-\kappa t_{2}$, then it is obvious that $T \in \mathcal{T}$. Therefore, the proof is complete from Theorem 2.4.

Corollary 2.7 Let $f: X \rightarrow X$ and $F: X \rightarrow C B(X)$ satisfy

$$
D(F x, F y) \leq \kappa \max \{d(f x, f y), d(f x, F x), d(f y, F y), 1 / 2[d(f x, F y)+d(f y, F x)]\}
$$

for some $\kappa$ with $0<\kappa<1$ and for all comparable elements $x, y$ of $X$. Also, assume that the following conditions are satisfied:

1. $F(X) \subseteq f(X)$ and $f(X)$ is closed.

2. If $f q \in F x$, then $x \preceq q$.

3. If $y_{n} \in F x_{n}$ is such that $y_{n} \rightarrow y=f p$, then $x_{n} \preceq p$ for all $n$.

Then $f v \in F v$. 
Remark 2.8 Corollary 2.6 extends Latif and Beg [2, Theorem 2.6], the result of Kaneko and Sessa [1] in a partially ordered set. Corollary 2.7 also extends the results of $[1-3,24]$ to a partially ordered set.

\section{Common fixed points}

In this section, we define $K-\preceq$ set-valued mappings on partially ordered metric spaces, and by using the definitions, we obtain the existence of common fixed points. Let $A$ and $B$ be two non-empty subsets of $(X, \preceq)$, the relations between $A$ and $B$ are denoted and defined as follows:

$$
A \prec_{1} B \text { : if for every } a \in A \text { there exists } b \in B \text { such that } a \preceq b \text {. }
$$

Definition 3.1 Let $M$ be a non-empty subset of $X$ and $F: M \rightarrow P(X)$. A mapping $F$ is said to be $K-\preceq$ set-valued if there exists $0 \leq \kappa<\frac{1}{2}$, and for any $x \in M, u_{x} \in F x$ there exists a $u_{y} \in F y$ with $u_{x} \preceq u_{y}$ such that

$$
d\left(u_{x}, u_{y}\right) \leq \kappa\left[d\left(x, u_{x}\right)+d\left(y, u_{y}\right)\right]
$$

for all $y \in M$ with $x \preceq y$.

For $K-\preceq$ set-valued mappings, we just required comparability of the elements, but order does not matter.

Theorem 3.2 Let $M$ be a non-empty closed subset of $X$ and $F: M \rightarrow C l(M)$ be a $K-\preceq$ set-valued mapping satisfying:

1. There exists $x_{0}$ in $M$ such that $\left\{x_{0}\right\} \prec_{1} F x_{0}$;

2. If $x_{n} \rightarrow x$ is a sequence in $M$ whose consecutive terms are comparable, then $x_{n} \preceq x$ for all $n$.

Then there exists $x \in M$ with $x \in F x$.

Proof Let $x_{0} \in M$. Then by assumption 1 , there exists $x_{1} \in F x_{0}$ such that $x_{0} \preceq x_{1}$. Now, since $F$ is a $K-\preceq$ set-valued mapping, there is $x_{2} \in F x_{1}$ with $x_{1} \preceq x_{2}$ such that

$$
d\left(x_{1}, x_{2}\right) \leq \kappa\left[d\left(x_{0}, x_{1}\right)+d\left(x_{1}, x_{2}\right)\right]
$$

which gives

$$
(1-\kappa) d\left(x_{1}, x_{2}\right) \leq \kappa d\left(x_{0}, x_{1}\right)
$$

and consequently

$$
d\left(x_{1}, x_{2}\right) \leq \frac{\kappa}{1-\kappa} d\left(x_{0}, x_{1}\right)
$$

Continuing in this way, we obtain a sequence $\left\{x_{n}\right\}$ whose consecutive terms are comparable and

$$
d\left(x_{n}, x_{n+1}\right) \leq\left[\frac{\kappa}{1-\kappa}\right]^{n} d\left(x_{0}, x_{1}\right) .
$$


Take $0 \leq h=\frac{\kappa}{1-\kappa}<1$, then we have

$$
d\left(x_{n}, x_{n+1}\right) \leq h^{n} d\left(x_{0}, x_{1}\right)
$$

Next, we will show that $\left\{x_{n}\right\}$ is a Cauchy sequence in $X$. Let $m>n$. Then

$$
\begin{aligned}
d\left(x_{n}, x_{m}\right) & \leq d\left(x_{n}, x_{n+1}\right)+d\left(x_{n+1}, x_{n+2}\right)+d\left(x_{n+2}, x_{n+3}\right)+\cdots+d\left(x_{m-1}, x_{m}\right) \\
& \leq\left[h^{n}+h^{n+1}+h^{n+2}+\cdots+h^{m-1}\right] d\left(x_{0}, x_{1}\right) \\
& =h^{n}\left[1+h+h^{2}+\cdots+h^{m-n-1}\right] d\left(x_{0}, x_{1}\right) \\
& =h^{n} \frac{1-h^{m-n}}{1-h} d\left(x_{0}, x_{1}\right) \\
& <\frac{h^{n}}{1-h} d\left(x_{0}, x_{1}\right)
\end{aligned}
$$

because $h \in(0,1), 1-h^{m-n}<1$.

Therefore, $d\left(x_{n}, x_{m}\right) \rightarrow 0$ as $n \rightarrow \infty$, which further implies that $\left\{x_{n}\right\}$ is a Cauchy sequence. So, there exists some point (say) $x$ in the complete metric space $X$ such that $x_{n} \rightarrow x$. By using assumption $2, x_{n} \preceq x$ for all $n$.

Further, since $M$ is closed, $x \in M$. Now we want to show that $x \in F x$.

Since $x_{n} \in F x_{n-1}$ with $x_{n-1} \preceq x_{n}$ also $x_{n} \preceq x$ for all $n$ and $\mathrm{F}$ is a $k-\preceq$ set-valued mapping, there exists $u_{n} \in F x$ with $x_{n} \preceq u_{n}$ such that

$$
d\left(x_{n}, u_{n}\right) \leq \kappa\left[d\left(x_{n-1}, x_{n}\right)+d\left(x, u_{n}\right)\right]
$$

Now

$$
d\left(x, u_{n}\right) \leq d\left(x, x_{n}\right)+d\left(x_{n}, u_{n}\right)
$$

and using (3.2), we have

$$
d\left(x, u_{n}\right) \leq \frac{1}{1-\kappa}\left[d\left(x, x_{n}\right)+\kappa d\left(x_{n-1}, x_{n}\right)\right]
$$

Letting $n \rightarrow \infty$, we obtain $u_{n} \rightarrow x$.

As $u_{n} \in F x$ and $F x$ is closed, so $x \in F x$.

Example 3.3 Let $M=\left\{(0,0),\left(0, \frac{1}{4}\right),\left(0, \frac{-1}{4}\right),\left(\frac{-1}{4}, \frac{1}{4}\right)\right\}$ be a subset of $X=R^{2}$ with usual order defined as follows: for $(u, v),(x, y) \in X,(u, v) \leq(x, y)$ if and only if $u \leq x, y \leq v$. Let $d$ be a metric on $X$ defined as follows:

$$
d(x, y)=d\left(\left(x_{1}, x_{2}\right),\left(y_{1}, y_{2}\right)\right):=\max \left\{\left|x_{1}-y_{1}\right|,\left|x_{2}-y_{2}\right|\right\}
$$

for all $x, y \in X$, so that $(X, d)$ is a complete metric space. Define $F: M \rightarrow C l(M)$ as follows:

$$
F(x, y)= \begin{cases}\{(0,0)\} & \text { if } x \geq y, \\ \left\{(0,0),\left(0, \frac{-1}{4}\right)\right\} & \text { if } x<y\end{cases}
$$

$\left(0, \frac{-1}{4}\right) \leq(0,0) \leq\left(0, \frac{1}{4}\right)$ and $\left(\frac{-1}{4}, \frac{1}{4}\right) \leq\left(0, \frac{1}{4}\right)$. 
Consider $x=\left(0, \frac{-1}{4}\right) \leq(0,0)=y$ for $u_{x}=(0,0) \in F x$, there exists $u_{y}=(0,0) \in F y$ such that $u_{x} \leq u_{y}$.

Next $0=d((0,0),(0,0)) \leq \kappa\left[d\left(\left(0, \frac{-1}{4}\right),(0,0)\right)+d((0,0),(0,0))\right]=\frac{\kappa}{4}$.

Similarly, for other comparable elements of $M$, one can see that $F$ is a $K-\leq$ set-valued mapping.

Further $(0,0) \in M$ is such that $\{0,0\} \prec_{1} F(0,0)$. Also, assumption 2 of Theorem 3.2 is satisfied and $(0,0)$ is the fixed point of $F$.

Theorem 3.4 Let $M$ be a non-empty closed subset of $X$ and $F_{n}: M \rightarrow C l(M)$ be a sequence of mappings satisfying the following:

(B): For any two mappings $F_{i}, F_{j}$ and for any $x \in M, u_{x} \in F_{i} x$, there exists a $u_{y} \in F_{j} y$ with $u_{x} \preceq u_{y}$ such that

$$
d\left(u_{x}, u_{y}\right) \leq \kappa\left[d\left(x, u_{x}\right)+d\left(y, u_{y}\right)\right]
$$

for all $y \in M$ with $x \leq y$ and for some $0 \leq \kappa<\frac{1}{2}$.

Assume that the following conditions also hold:

1. For each $x_{0} \in M,\left\{x_{0}\right\} \prec_{1} F_{1} x_{0}$.

2. If $x_{n} \rightarrow x$ is a sequence in $X$ whose consecutive terms are comparable, then $x_{n} \preceq x$ for all $n$.

Then there exists $x \in M$ with $x \in \cap F_{n} x$.

Proof Let $x_{0} \in M$. Then by assumption 1 , there exists $x_{1} \in F_{1} x_{0}$ such that $x_{0} \preceq x_{1}$. Now, using (B), there is $x_{2} \in F_{2} x_{1}$ with $x_{1} \preceq x_{2}$ such that

$$
d\left(x_{1}, x_{2}\right) \leq \kappa\left[d\left(x_{0}, x_{1}\right)+d\left(x_{1}, x_{2}\right)\right]
$$

which gives

$$
d\left(x_{1}, x_{2}\right) \leq \frac{\kappa}{1-\kappa} d\left(x_{0}, x_{1}\right)
$$

Next, for this $x_{2}$, there exists $x_{3} \in F_{3} x_{2}$ with $x_{2} \preceq x_{3}$ such that

$$
d\left(x_{2}, x_{3}\right) \leq \kappa\left[d\left(x_{1}, x_{2}\right)+d\left(x_{2}, x_{3}\right)\right]
$$

Using (3.3), we obtain

$$
d\left(x_{2}, x_{3}\right) \leq\left[\frac{\kappa}{1-\kappa}\right]^{2} d\left(x_{0}, x_{1}\right)
$$

Continuing in this way, we obtain a sequence $x_{n}$ whose consecutive terms are comparable and

$$
d\left(x_{n}, x_{n+1}\right) \leq\left[\frac{\kappa}{1-\kappa}\right]^{n} d\left(x_{0}, x_{1}\right) .
$$

Take $0 \leq h=\frac{\kappa}{1-\kappa}<1$, then we have

$$
d\left(x_{n}, x_{n+1}\right) \leq h^{n} d\left(x_{0}, x_{1}\right) .
$$


Therefore, $\left\{x_{n}\right\}$ is a Cauchy sequence in a complete metric space $X$, so $x_{n} \rightarrow x$. By using assumption $2, x_{n} \preceq x$ for all $n$.

Further, since $M$ is closed, $x \in M$. Let $F_{m}$ be any arbitrary member of $F_{n}$. Now, since $x_{n} \in F_{n} x_{n-1}$ with $x_{n-1} \preceq x_{n}$. Also, $x_{n} \preceq x$ for all $n$. By using (B), there exists $u_{n} \in F_{m} x$ such that

$$
d\left(x_{n}, u_{n}\right) \leq \kappa\left[d\left(x_{n-1}, x_{n}\right)+d\left(x, u_{n}\right)\right]
$$

Now

$$
d\left(x, u_{n}\right) \leq d\left(x, x_{n}\right)+d\left(x_{n}, u_{n}\right)
$$

which gives

$$
d\left(x, u_{n}\right) \leq \frac{1}{1-\kappa}\left[d\left(x, x_{n}\right)+\kappa d\left(x_{n-1}, x_{n}\right)\right]
$$

Letting $n \rightarrow \infty$, we have $u_{n} \rightarrow x$.

As $u_{n} \in F_{m} x$ and $F_{m} x$ is closed, so $x \in F_{m} x$, i.e., $x \in F_{n} x$.

Remark 3.5 Theorems 3.2 and 3.4 improve/extend [2, Theorems 4.1 and 4.2].

\section{Competing interests}

The authors declare that they have no competing interests.

\section{Authors' contributions}

IB gave the idea and ARB wrote the initial draft. Both authors read and agreed upon the draft and finalized the manuscript. Correspondence was mainly done by IB. All authors read and approved the final manuscript.

\section{Author details}

${ }^{1}$ Centre for Applicable Mathematics and Statistics, University of Central Punjab, Lahore, Pakistan. ${ }^{2}$ Department of Mathematics, University of Engineering and Technology, Lahore, Pakistan.

\section{Acknowledgements}

The present version of the paper owes much to the precise and kind remarks of the learned referees.

Received: 26 July 2012 Accepted: 29 November 2012 Published: 18 December 2012

\section{References}

1. Kaneko, H: Single-valued and multi-valued $f$-contractions. Boll. Unione Mat. Ital., A 6(4), 29-33 (1985)

2. Latif, A, Beg, I: Geometric fixed points for single and multivalued mappings. Demonstr. Math. 30(4), 791-800 (1997)

3. Kaneko, H, Sessa, S: Fixed point theorems for compatible multi-valued and single-valued mappings. Int. J. Math. Math. Sci. 12(2), 252-262 (1989)

4. Karapinar, E: Ćirić types non-unique fixed point theorems on partial metric spaces. J. Nonlinear Sci. Appl. 5, 74-83 (2012)

5. Lakshmikantham, V, Ćirić, L: Coupled fixed point theorems for nonlinear contractions in partially ordered metric spaces. Nonlinear Anal. 70(12), 4341-4349 (2009)

6. Nashine, HK: New fixed point theorems for mappings satisfying generalized weakly contractive condition with weaker control functions. Ann. Pol. Math. 104, 109-119 (2012)

7. Nieto, JJ, Rodríguez-López, R: Contractive mapping theorems in partially ordered sets and applications to ordinary differential equations. Order 22, 223-239 (2005)

8. Nieto, JJ, Rodríguez-López, R: Existence and uniqueness of fixed point in partially ordered sets and applications to ordinary differential equations. Acta Math. Sin. Engl. Ser. 23, 2205-2212 (2007)

9. Nieto, JJ, Pouso, RL, Rodríguez-López, R: Fixed point theorems in ordered abstract spaces. Proc. Am. Math. Soc. 135, 2505-2517 (2007)

10. Nieto, JJ, Rodríguez-López, R: Existence of extremal solutions for quadratic fuzzy equations. Fixed Point Theory Appl. 2005(3), 321-342 (2005)

11. Ran, ACM, Reurings, MCB: A fixed point theorem in partially ordered sets and some applications to matrix equations. Proc. Am. Math. Soc. 132, 1435-1443 (2004) 
12. O'Regan, D, Petrusel, A: Fixed point theorems for generalized contraction in ordered metric spaces. J. Math. Anal. Appl. 192, 655-666 (2007)

13. Cabada, A, Nieto, JJ: Fixed points and approximate solutions for nonlinear operator equations. J. Comput. Appl. Math. $113,17-25(2000)$

14. Drici, Z, McRae, FA, Devi, JV: Fixed point theorems in partially ordered metric space for operators with PPF dependence. Nonlinear Anal. 67, 641-647 (2007)

15. Petrusel, A, Rus, IA: Fixed point theorems in ordered L-spaces. Proc. Am. Math. Soc. 134, 411-418 (2005)

16. Beg, I, Butt, AR: Fixed point for set valued mappings satisfying an implicit relation in partially ordered metric spaces. Nonlinear Anal. 71, 3699-3704 (2009)

17. Beg, I, Butt, AR: Fixed point for weakly compatible mappings satisfying an implicit relation in partially ordered metric spaces. Carpath. J. Math. 25, 1-12 (2009)

18. Beg, I, Butt, AR: Common fixed point for generalized set valued contractions satisfying an implicit relation in partially ordered metric spaces. Math. Commun. 15, 65-75 (2010)

19. Beg, I, Butt, AR, Radojević, S: Contraction principle for set valued mappings on a metric space with a graph. Comput. Math. Appl. 60, 1214-1219 (2010)

20. Beg, I, Nashine, HK: End-point results for multivalued mappings in partially ordered metric spaces. Int. J. Math. Math. Sci. 2012, Article ID 580250 (2012)

21. Granas, A, Dugundji, J: Fixed Point Theory. Springer, New York (2003)

22. Tarski, A: A lattice theoretical fixed point theorem and its application. Pac. J. Math. 5, 285-309 (1955)

23. Kannan, R: Some results on fixed points. Bull. Calcutta Math. Soc. 60, 71-76 (1968)

24. Singh, SL, Kulshrestha, C: Coincidence theorems in metric spaces. Indian J. Phys. Nat. Sci., Ser. B 2, 19-22 (1982)

25. Assad, NA, Kirk, WA: Fixed point theorems for set valued mappings of contractive type. Pac. J. Math. 43, 533-562 (1972)

26. Altun, I: Fixed point and homotopy results for multivalued maps satisfying an implicit relation. J. Fixed Point Theory Appl. 9, 125-134 (2011)

27. Kamran, T: Coincidence and fixed points for hybrid strict contractions. J. Math. Anal. Appl. 299, 235-241 (2004)

doi:10.1186/1687-1812-2012-229

Cite this article as: Beg and Butt: Common fixed point and coincidence point of generalized contractions in ordered metric spaces. Fixed Point Theory and Applications 2012 2012:229.

\section{Submit your manuscript to a SpringerOpen ${ }^{\ominus}$ journal and benefit from:}

- Convenient online submission

Rigorous peer review

- Immediate publication on acceptance

- Open access: articles freely available online

- High visibility within the field

- Retaining the copyright to your article 